\title{
All trials must be registered and the results published
}

\author{
Academics and non-commercial funders are just as guilty as industry
}

\author{
Iain Chalmers coordinator ${ }^{1}$, Paul Glasziou professor ${ }^{2}$, Fiona Godlee editor in chief $^{3}$ \\ ${ }^{1}$ James Lind Initiative, Oxford OX2 7LG, UK ; ${ }^{2}$ Centre for Research in Evidence-Based Practice, Faculty of Health Sciences, Bond University, Gold \\ Coast, QLD, Australia; ${ }^{3} \mathrm{BMJ}$, London, UK
}

\begin{abstract}
Biased under-reporting of research has been documented for well over two decades and the evidence for it is now overwhelming. ${ }^{1-4}$ Under-reporting is research misconduct and has serious consequences. ${ }^{56}$ It leads to overestimates of the benefits of treatments and underestimates of their harmful effects. ${ }^{7}$ Because of this it puts patients at risk and wastes healthcare resources.
\end{abstract}

Much of the criticism has focused on commercially funded trials, and justifiably so. There is clear and consistent evidence of under-reporting and manipulation of the scientific literature by the drug and devices industries, ${ }^{4}$ and industry sponsors most of the world's clinical trials. But under-reporting is not confined to commercially sponsored trials. Indeed, early examples of failure to publish negative results came from academia. ${ }^{5}$

Nor has academia been any better than industry at cleaning up its act in the intervening decades. Because of trial registration, we can now estimate the magnitude and describe some of the characteristics of under-reporting of clinical trials. Only around half of all registered trials have published at least some of their results, and this level of under-reporting affects most types of trial: early and late phase, large and small, national and international, commercial and non-commercial (figure). ${ }^{9}$

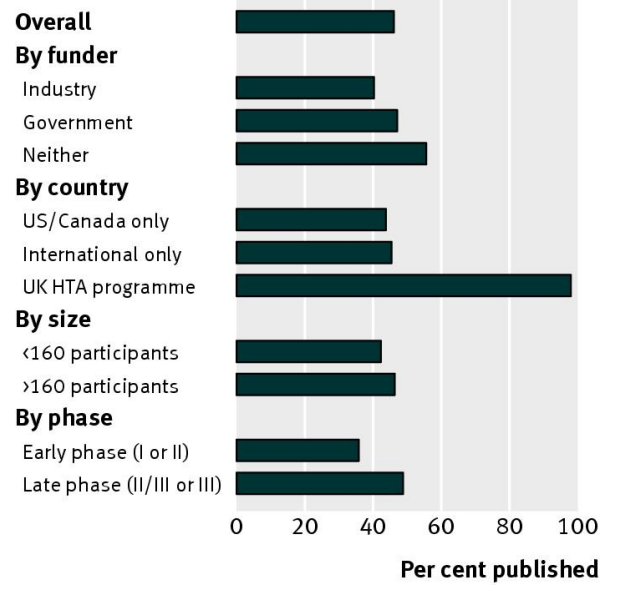

Proportion of clinical trials registered by 1999 and published by $2007^{9}$
This matters because participants in clinical trials assume that they are contributing to the advancement of medical knowledge; non-publication of study results negates this reasonable assumption and betrays those who have volunteered.

Non-publication also matters because failure to publish all the results from clinical trials distorts the evidence base for clinical decisions. In a Personal View published in the $B M J$ eight years ago, the clinical epidemiologist Alessandro Liberati protested that the unpublished results of clinical trials could have informed his choices as a patient with multiple myeloma. "Why was I forced to make my decision knowing that information was somewhere but not available? Was the delay because the results were less exciting than expected? Or because in the evolving field of myeloma research there are now new exciting theories (or drugs) to look at? How far can we tolerate the butterfly behaviour of researchers, moving on to the next flower well before the previous one has been fully exploited?"10 Just over a year ago, Liberati died from the complications of his disease, still waiting for researchers to publish information relevant to his treatment choices.

Many academic trials have failed to report their findings, including important trials supported by major funders. For example, a large trial of adenoidectomy funded by the UK's Medical Research Council remained unpublished for more than a decade after it was concluded. The study has now at last been reported. ${ }^{11}$ And this week the $B M J$ reports on the failure of US academics to publish protocol defined follow-up data from a trial of sentinel node biopsy in malignant melanoma. ${ }^{12}$

What can explain this failure to publish academic trials? Journals have been blamed for a bias towards accepting positive results, and some of the blame does lie with them. But the evidence indicates that the principal culprits are authors and research sponsors for not submitting reports for publication. ${ }^{13}$ Financial conflict of interest is well understood as a motive for suppression of unfavourable results from commercially sponsored trials. But what are the motives of authors and sponsors of non-commercial trials? Authors admit failure to write up and submit their results, ${ }^{14}$ and anecdotes suggest a range of reasons, such as losing interest or moving on to new institutions and 
projects, poor organisation, inadequate resources, writer's block, or unwillingness to accept the results of a trial owing to intellectual or reputational investment in the outcome. Despite the billions of pounds wasted, there has been too little systematic effort to monitor the extent of non-publication, let alone investigate the reasons for it.

The responsibilities of authors are clear: the Helsinki Declaration leaves no room for ambiguity. It states that, "Authors have a duty to make publicly available the results of their research on human subjects and are accountable for the completeness and accuracy of their reports ... Negative and inconclusive as well as positive results should be published or otherwise made publicly available."15

But authors' behaviour is unlikely to change without firm action from those who give ethics approval, institutional hosting, and funding support for trials. Research ethics committees were challenged long ago to behave ethically by ensuring that results of trials were published, ${ }^{16}$ yet these committees have been noticeable by their absence among those exposing under-reporting of clinical trials and taking steps to tackle the problem. It is clear from the figure that academic institutions and funders of research have similarly failed in their responsibilities. There are exceptions, however: the figure also shows that $98 \%$ of the studies funded by the National Institute for Health Research Health Technology Assessment Programme have led to the publication of full reports (Ruairidh Milne, personal communication). The programme has achieved this by holding back a proportion of the research grant until a report has been submitted for publication, by chasing authors on a regular basis, and by providing a publication vehicle-Health Technology Assessment — for all trials.

This shows what can and should be done. Information made public through trial registration means that research funders and institutions that continue to under-report clinical trials can now be identified. Patients who are invited to participate in trials should consider the track record of the institutions and funders concerned and refuse to participate unless they receive written assurance that the full study results will be made publicly available and freely accessible (box).
A campaign to ensure that all trials are registered and their results published, or otherwise made publicly available, is launched this week (alltrials.net). We invite all $B M J$ readers to sign the campaign's petition.

Competing interests: We have read and understood the BMJ Group policy on declaration of interests and we have no relevant interests to declare.

Provenance and peer review: Commissioned; not externally peer reviewed.

1 Chalmers I. Proposal to outlaw the term "negative trial." BMJ 1985;290:1002.

Dickersin K, Chalmers I. Recognising, investigating and dealing with incomplete and biased reporting of clinical research: from Francis Bacon to the World Health Organisation. 2010. www.jameslindlibrary.org/illustrating/articles/recognising-investigating-and-dealing with-incomplete-and-biase.

3 Song F, Parekh S, Hooper L, Loke YK, Ryder JJ, Sutton AJ, et al. Dissemination and publication of research findings: an updated review of related biases. Health Technol Assess 2010;14(8).

4 Goldacre B. Bad pharma. Fourth Estate, 2012

5 Chalmers I. Underreporting research is scientific misconduct. JAMA 2010;263:1405-8.

6 A consensus statement on research misconduct in the UK. BMJ 2012;344:e1111.

7 McGauran N, Wieseler B, Kreis J, Schüler Y-B, Kölsch H, Kaiser T. Reporting bias in medical research-a narrative review. Trials 2010;11:37.

8 Simes RJ. Publication bias: the case for an international registry of clinical trials. J Clin Oncol 1986;4:1529-41.

9 Ross JS, Mulvey GK, Hines EM, Nissen SE, Krumholz HM. Trial publication after registration in clinicaltrials.gov: a cross-sectional analysis. PLoS Med 2009;6:e1000144. 10 Liberati A. An unfinished trip through uncertainties. BMJ 2004;328:531.

11 MRC Multicentre Otitis Media Study Group. Adjuvant adenoidectomy in persistent bilateral otitis media with effusion: hearing and revision surgery outcomes through 2 years in the TARGET randomised trial. Clin Otolaryngol 2012;37:107-16.

12 Torjesen I. Sentinel node biopsy for melanoma: is it worth it? BMJ 2013;346:e8645

13 Chalmers I, Dickersin K. Biased under-reporting of research reflects biased under-submission more than biased editorial rejection. F1000 Res 2012;1:69.

14 Smyth RMD, Kirkham JJ, Jacoby A, Altman DG, Gamble C, Williamson PR. Frequency and reasons for outcome reporting bias in clinical trials: interviews with trialists. $B M J$ 2012;342:c7153.

15 World Medical Association. Ethical principles for medical research involving human subjects. 2008. www.wma.net/en/30publications/10policies/b3/.

16 Savulescu J, Chalmers I, Blunt J. Are research ethics committees behaving unethically? Some suggestions for improving performance and accountability. BMJ 1996:313:1390-3.

17 Evans I, Thornton H, Chalmers I, Glasziou P. Testing treatments. Pinter and Martin, 2011. www.testingtreatments.org.

Cite this as: BMJ 2013;346:f105

(c) BMJ Publishing Group Ltd 2013 


\section{Advice to patients invited to participate in a clinical trial ${ }^{17}$}

Agree to participate in a clinical trial only if: (1) the study protocol has been registered and made publicly available; (2) the protocol refers to systematic reviews of existing evidence showing that the trial is justified; and (3) you receive a written assurance that the full study results will be published and sent to all participants who indicate that they wish to receive them. 